Bull. Korean Math. Soc. 52 (2015), No. 3, pp. 881-893

http://dx.doi.org/10.4134/BKMS.2015.52.3.881

\title{
FIXED POINT THEOREMS OF WEAKLY MONOTONE PREŠIĆ TYPE MAPPINGS IN ORDERED CONE METRIC SPACES
}

\author{
Mohammad Saeed Khan, Satish Shukla, and Shin Min Kang
}

\begin{abstract}
In this paper, we introduce the weakly monotone Prešić type mappings in product spaces when the underlying space is an ordered cone metric space. Some fixed point results for such mappings are also proved which generalize and unify several known results in metric and cone metric spaces with normal cone. The results are supported by examples.
\end{abstract}

\section{Introduction}

In 1905, the famous French mathematician Fréchet [4] introduced the concept of a metric space. According to the need, several mathematicians generalized this concept in various directions. In 1934, a Serbian mathematician Kurepa $[10,11]$ introduced more abstract metric spaces, in which the metric takes values in an ordered vector space. For some more similar generalizations the reader is referred to [12, 23, 29].

Recently, Huang and Zhang [6] reintroduced such spaces under the name of cone metric spaces, where every pair of elements is assigned to an element of a Banach space equipped with a cone which induces a natural partial order. In the same work, they investigated the convergence in cone metric spaces, introduced the notion of their completeness, and proved some fixed point theorems for mappings satisfying various contractive conditions on these spaces.

On the other hand, when considering the convergence of some particular sequences, Prešić [20] generalized the Banach contraction principle [1] in product spaces and proved the following theorem.

Theorem 1.1. Let $(X, d)$ be a complete metric space, $k$ a positive integer and $T: X^{k} \rightarrow X$ be a mapping satisfying the following contractive type condition:

$$
\begin{aligned}
& d\left(T\left(x_{1}, x_{2}, \ldots, x_{k}\right), T\left(x_{2}, x_{3}, \ldots, x_{k+1}\right)\right) \\
\leq & q_{1} d\left(x_{1}, x_{2}\right)+q_{2} d\left(x_{2}, x_{3}\right)+\cdots+q_{k} d\left(x_{k}, x_{k+1}\right)
\end{aligned}
$$

Received March 26, 2014; Revised July 7, 2014.

2010 Mathematics Subject Classification. 54H25, $47 \mathrm{H} 10$.

Key words and phrases. Prešić type mapping, weakly monotone Prešić type mapping, ordered cone metric space, fixed point. 
for every $x_{1}, x_{2}, \ldots, x_{k+1} \in X$, where $q_{1}, q_{2}, \ldots, q_{k}$ are nonnegative constants such that $q_{1}+q_{2}+\cdots+q_{k}<1$. Then there exists a unique point $x \in X$ such that $T(x, x, \ldots, x)=x$. Moreover, if $x_{1}, x_{2}, \ldots, x_{k}$ are arbitrary points in $X$ and for $n \in \mathbb{N}, x_{n+k}=T\left(x_{n}, x_{n+1}, \ldots, x_{n+k-1}\right)$, then the sequence $\left\{x_{n}\right\}$ is convergent and $\lim x_{n}=T\left(\lim x_{n}, \lim x_{n}, \ldots, \lim x_{n}\right)$.

The result of Prešić is generalized by several authors (see, e.g., [8, 17, 18, 19, 24, 25, 26, 27, 28]). George et al. [5], Khan and Samanipour [9], and Malhotra et al. [14] proved the cone metric version of result of Prešić.

The existence of fixed point in partially ordered sets endowed with a metric was first investigated by Nieto and López [16], and Ran and Reurings [21]. They proved the ordered version of Banach contraction principle. Also, they considered some applications of fixed point results in ordered spaces.

Recently, Luong and Thuan [13], Malhotra et al. [14], and Shukla and Radenović [26] proved the ordered version of theorem of Prešić in cone metric, metric and partial metric spaces. In all these papers, the Prešić type mapping was assumed to be "monotone".

In this paper, we introduce the notion of weakly monotone Prešić type mappings (which need not be monotone) in the setting of cone metric spaces with normal cone and use a more general contractive condition on mapping to prove the existence and uniqueness of fixed point of such mappings in ordered cone metric spaces. Our results generalize and extend the results of $[6,9,14,21]$ in ordered cone metric spaces with normal cone. Some examples are given which validate our results.

\section{Preliminaries}

We need the following definitions and results, consistent with [3] and [6].

Definition 2.1 ([6]). Let $E$ be a real Banach space and $P$ be a subset of $E$. The set $P$ is called a cone if

(i) $P$ is closed, nonempty and $P \neq\left\{0_{\mathrm{E}}\right\}$, where $0_{\mathrm{E}}$ is the zero vector of $E$;

(ii) $a, b \in \mathbb{R}, a, b \geq 0, x, y \in P \Rightarrow a x+b y \in P$;

(iii) $x \in P$ and $-x \in P \Rightarrow x=0_{\mathrm{E}}$.

Given a cone $P \subset E$, we define a partial ordering " $\preceq_{\mathrm{P}}$ " with respect to $P$ by $x \preceq_{\mathrm{P}} y$ if and only if $y-x \in P$. We write $x \prec_{\mathrm{P}} y$ to indicate that $x \preceq_{\mathrm{P}} y$ but $x \neq y$. While $x \ll_{\mathrm{P}} y$ if and only if $y-x \in P^{0}$, where $P^{0}$ denotes the interior of $P$.

Let $P$ be a cone in a real Banach space $E$, then $P$ is called normal, if there exist a constant $K>0$ such that for all $x, y \in E$,

$$
0_{\mathrm{E}} \preceq_{\mathrm{P}} x \preceq_{\mathrm{P}} y \text { implies }\|x\| \leq K\|y\| .
$$

The least positive number $K$ satisfying the above inequality is called the normal constant of $P$.

Definition $2.2([6])$. Let $X$ be a nonempty set, $E$ be a real Banach space with cone $P$. Suppose that the mapping $d: X \times X \rightarrow E$ satisfies: 
1. $0_{\mathrm{E}} \preceq_{\mathrm{P}} d(x, y)$ for all $x, y \in X$ and $d(x, y)=0_{\mathrm{E}}$ if and only if $x=y$;

2. $d(x, y)=d(y, x)$ for all $x, y \in X$;

3. $d(x, y) \preceq_{\mathrm{P}} d(x, z)+d(y, z)$ for all $x, y, z \in X$.

Then " $d$ " is called a cone metric on $X$, and $(X, d)$ is called a cone metric space. If the underlying cone is normal, then $(X, d)$ is called a normal cone metric space.

The concept of cone metric space is more general than that of a metric space, because each metric space is a cone metric space with $E=\mathbb{R}$ and $P=[0,+\infty)$.

The following remark will be useful in the sequel.

Remark $2.1([7])$. Let $P$ be a cone in a real Banach space $E$ with zero vector $0_{\mathrm{E}}$ and $a, b, c \in P$. Then

1. If $a \preceq_{\mathrm{P}} b$ and $b \ll_{\mathrm{P}} c$, then $a \ll_{\mathrm{P}} c$.

2. If $a \ll_{\mathrm{P}} b$ and $b \ll_{\mathrm{P}} c$, then $a \ll_{\mathrm{P}} c$.

3. If $0_{\mathrm{E}} \preceq_{\mathrm{P}} u \ll_{\mathrm{P}} c$ for each $c \in P^{0}$, then $u=0_{\mathrm{E}}$.

4. If $c \in P^{0}$ and $a_{n} \rightarrow 0_{\mathrm{E}}$, then there exist $n_{0} \in \mathbb{N}$ such that, for all $n>n_{0}$ we have $a_{n} \ll_{\mathrm{P}} c$.

5. If $0_{\mathrm{E}} \preceq_{\mathrm{P}} a_{n} \preceq_{\mathrm{P}} b_{n}$ for each $n$ and $a_{n} \rightarrow a, b_{n} \rightarrow b$, then $a \preceq_{\mathrm{P}} b$.

6 . If $a \preceq_{\mathrm{P}} \lambda a$ where $0 \leq \lambda<1$, then $a=0_{\mathrm{E}}$.

Definition 2.3 $([6])$. Let $(X, d)$ be a cone metric space. Let $\left\{x_{n}\right\}$ be a sequence in $X$ and $x \in X$.

1. If for every $c \in E$ with $0 \ll_{\mathrm{P}} c$ (or equivalently $c \in P^{0}$ ) there is a positive integer $n_{0}$ such that, $d\left(x_{n}, x\right) \ll_{\mathrm{P}} c$ for all $n>n_{0}$, then the sequence $\left\{x_{n}\right\}$ is said to be convergent at $x$. We denote this by $\lim _{n \rightarrow \infty} x_{n}=x$ or $x_{n} \rightarrow x$ as $n \rightarrow \infty$.

2. If for every $c \in E$ with $0 \ll_{\mathrm{P}} c$ there is a positive integer $n_{0}$ such that, $d\left(x_{n}, x_{m}\right) \ll_{\mathrm{P}} c$ for all $n, m>n_{0}$, then the sequence $\left\{x_{n}\right\}$ is called a Cauchy sequence in $X$.

3. $(X, d)$ is called a complete cone metric space if every Cauchy sequence in $X$ is convergent in $X$.

Definition 2.4. Let $(X, d)$ be a cone metric space, $k$ be a positive integer and $T: X^{k} \rightarrow X$ be a mapping. If $T(x, x, \ldots, x)=x$, then $x \in X$ is called a fixed point of $T$.

Lemma $2.2([6])$. Let $(X, d)$ be a cone metric space, $P$ be a normal cone with normal constant $K$. Let $\left\{x_{n}\right\}$ and $\left\{y_{n}\right\}$ be two sequences in $X$.

(a) $\left\{x_{n}\right\}$ is Cauchy sequence if and only if $d\left(x_{n}, x_{m}\right) \rightarrow 0_{E}$ as $n \rightarrow \infty$.

(b) If $x_{n} \rightarrow x, y_{n} \rightarrow y$ as $n \rightarrow \infty$, then $d\left(x_{n}, y_{n}\right) \rightarrow d(x, y)$ as $n \rightarrow \infty$.

Following definitions can be found in $[13,14,15]$.

Let $E, B$ be two real Banach spaces and $P, C$ be normal cones in $E$ and $B$, respectively. Let " $\preceq_{\mathrm{P}}$ " and " $\preceq_{\mathrm{C}}$ " be the partial orderings induced by $P$ and $C$ in $E$ and $B$, respectively. Let $\phi: P \rightarrow C$ be a function satisfying:

(i) if $a, b \in P$ with $a \preceq_{\mathrm{P}} b$, then $\phi[a] \preceq_{\mathrm{C}} \alpha \phi[b]$ for some positive real numbers $\alpha$; 
(ii) $\phi[a+b] \preceq_{\mathrm{C}} \phi[a]+\phi[b]$ for all $a, b \in P$;

(iii) $\phi$ is sequentially continuous, that is, if $a_{n}, a \in P$ and $a_{n} \rightarrow a$ as $n \rightarrow \infty$, then $\phi\left[a_{n}\right] \rightarrow \phi[a]$ as $n \rightarrow \infty$;

(iv) if $\phi\left[a_{n}\right] \rightarrow 0_{\mathrm{B}}$ as $n \rightarrow \infty$, then $a_{n} \rightarrow 0_{\mathrm{E}}$ as $n \rightarrow \infty$, where $0_{\mathrm{E}}$ and $0_{\mathrm{B}}$ are the zero vectors of $E$ and $B$, respectively.

We denote the set of all such functions by $\Phi(P, C)$, that is, $\phi \in \Phi(P, C)$ if $\phi$ satisfies all the above properties. From the definition it is clear that $\phi[a]=0_{\mathrm{B}}$ if and only if $a=0_{\mathrm{E}}$.

Example 2.1. Let $E$ be the real vector space defined by

$$
E=\left\{a x+b: a, b \in \mathbb{R}, x \in\left[\frac{1}{2}, 1\right]\right\}
$$

with supremum norm and $P=\{a x+b \in E: a \leq 0, b \geq 0\}$. Then $P$ is a normal cone with normal constant $K>2$ (see [22]). Let $B=\mathbb{R}^{2}$ with Euclidean norm and $C=\{(a, b): a \geq 0, b \geq 0\}$. Then $C$ is a normal cone with normal constant $K=1$. Define $\phi: P \rightarrow C$ by $\phi[a x+b]=(-a, b)$ for all $a x+b \in P$. Then $\phi \in \Phi(P, C)$ with $\alpha=1$.

For some more examples of function $\phi$ we refer to [15].

In the following we always suppose that $E, B$ are two real Banach spaces, $P$ and $C$ are normal cones in $E$ and $B$, respectively, " $\preceq_{\mathrm{P}}$ " and " $\preceq_{\mathrm{C}}$ " are partial orderings in $E$ and $B$ with respect to $P$ and $C$, respectively, and $0_{\mathrm{E}}$ and $0_{\mathrm{B}}$ are the zero vectors of $E$ and $B$, respectively.

Consider a function $\psi: B^{k} \rightarrow B$ such that:

1. $\psi\left(x_{1}, x_{2}, \ldots, x_{i-1}, x_{i}, x_{i+1}, \ldots, x_{k}\right) \preceq_{\mathrm{C}} \psi\left(x_{1}, x_{2}, \ldots, x_{i+1}, y_{i}, x_{i+1}, \ldots, x_{k}\right)$ whenever $x_{i} \preceq_{\mathrm{C}} y_{i}$ for any $i$ with $1 \leq i \leq k$,

2. $\psi(x, x, \ldots, x) \preceq_{\mathrm{C}} x$ for all $x \in B$.

We denote the set of all such functions by $\Psi(B)$, that is, $\psi \in \Psi(B)$ if $\psi$ satisfies all the above properties.

Example 2.2. Let $B=\mathbb{R}^{2}$ with Euclidian norm and $C=\{(a, b): a \geq 0, b \geq$ 0 . Then $C$ is a normal cone in $B$ with normal constant $K=1$. Define $\psi$ : $B^{k} \rightarrow B$ by $\psi\left(x_{1}, x_{2}, \ldots, x_{k}\right)=\sum_{i=1}^{k} \alpha_{i} x_{i}$ for all $x_{1}, x_{2}, \ldots, x_{k} \in B$, where $\alpha_{i}$ are nonnegative constants such that $\sum_{i=1}^{k} \alpha_{i}<1$. Then $\psi \in \Psi(B)$.

Now we define weakly monotone mappings on product spaces when the underlying spaces is a cone metric space with a partial order.

Definition 2.5. Let a nonempty set $X$ is equipped with a partial order " $\sqsubseteq$ " such that $(X, d)$ is a cone metric space, then $(X, \sqsubseteq, d)$ is called an ordered cone metric space. A sequence $\left\{x_{n}\right\}$ in $X$ is said to be nondecreasing with respect to " $\sqsubseteq$ " if $x_{1} \sqsubseteq x_{2} \sqsubseteq \cdots \sqsubseteq x_{n} \sqsubseteq \cdots$. Let $k$ be a positive integer and $T: X^{k} \rightarrow X$ be a mapping, then $T$ is said to be nondecreasing with respect to " $\sqsubseteq$ " if for any finite nondecreasing sequence $\left\{x_{n}\right\}_{n=1}^{k+1}$ we have $T\left(x_{1}, x_{2}, \ldots, x_{k}\right) \sqsubseteq T\left(x_{2}, x_{3}, \ldots, x_{k+1}\right) . \quad T$ is said to be nonincreasing with 
respect to " $\sqsubseteq$ " if for any finite nondecreasing sequence $\left\{x_{n}\right\}_{n=1}^{k+1}$ we have $T\left(x_{2}, x_{3}, \ldots, x_{k+1}\right) \sqsubseteq T\left(x_{1}, x_{2}, \ldots, x_{k}\right) . T$ is said to be monotone if it is nondecreasing or nonincreasing. The elements $x, y \in X$ are said to be comparable and we write $x \asymp y$ if $x \sqsubseteq y$ or $y \sqsubseteq x$. The sequence $\left\{x_{n}\right\}$ in $X$ is said to be weakly monotone if $x_{n} \asymp x_{n+1}$ for all $n \in \mathbb{N}$. T is said to be weakly monotone with respect to " $\sqsubseteq$ " if for any finite weakly monotone sequence $\left\{x_{n}\right\}_{n=1}^{k+1}$ we have $T\left(x_{1}, x_{2}, \ldots, x_{k}\right) \asymp T\left(x_{2}, x_{3}, \ldots, x_{k+1}\right)$.

It is clear that every nondecreasing or nonincreasing mapping is weakly monotone but converse need not be true in general. Following example shows that a weakly monotone mapping may not be a monotone mapping.

Example 2.3. Let $X=\{1,2,3,4\}$ and let the order relation on $X$ be defined by

$$
\sqsubseteq=\{(1,1),(2,2),(3,3),(4,4),(3,1),(1,4),(3,4)\} .
$$

For $k=2$, define a mapping $T: X^{2} \rightarrow X$ by

$$
T(x, y)= \begin{cases}\min \{x, y\} & \text { if } x, y \in X \text { with } x \neq y \\ x & \text { if } x=y \in\{1,2,3\} \\ 3 & \text { if } x=y=4 .\end{cases}
$$

Then $T$ is neither a nondecreasing nor a nonincreasing mapping. Indeed, $1 \sqsubseteq$ $4 \sqsubseteq 4$ but $T(1,4)=1 \nsubseteq 3=T(4,4)$, therefore $T$ is not nondecreasing. Again, $3 \sqsubseteq 3 \sqsubseteq 1$ but $T(3,1)=1 \nsubseteq 3=T(3,3)$, therefore $T$ is not nonincreasing. Now by a careful observation one can see that $T$ is weakly monotone mapping.

Definition 2.6. Let $(X, \sqsubseteq, d)$ be an ordered cone metric space with cone $P, k$ a positive integer and $T: X^{k} \rightarrow X$ be a mapping. Then the mapping $T$ is called a weakly ordered Prešic type contraction if there exist nonnegative constants $\alpha_{i}$ such that $\sum_{i=1}^{k} \alpha_{i}<1$ such that

$$
d\left(T\left(x_{1}, x_{2}, \ldots, x_{k}\right), T\left(x_{2}, x_{3}, \ldots, x_{k+1}\right)\right) \preceq_{\mathrm{P}} \sum_{i=1}^{k} \alpha_{i} d\left(x_{i}, x_{i+1}\right)
$$

for all $x_{1}, x_{2}, \ldots, x_{k+1} \in X$ with $x_{1} \asymp x_{2} \asymp \cdots \asymp x_{k+1}$.

Definition 2.7. Let $(X, \sqsubseteq, d)$ be an ordered cone metric space with cone $P$, $k$ a positive integer and $T: X^{k} \rightarrow X$ be a mapping. Then the mapping $T$ is called a weakly ordered Prešić-Cirić type contraction (see [2]) if there exists $\lambda \in(0,1)$ such that:

$$
\begin{aligned}
& d\left(T\left(x_{1}, x_{2}, \ldots, x_{k}\right), T\left(x_{2}, x_{3}, \ldots, x_{k+1}\right)\right) \\
& \preceq_{\mathrm{P}} \lambda \max \left\{d\left(x_{i}, x_{i+1}\right), 1 \leq i \leq k\right\}
\end{aligned}
$$

for all $x_{1}, x_{2}, \ldots, x_{k+1} \in X$ with $x_{1} \asymp x_{2} \asymp \cdots \asymp x_{k+1}$, provided that the maximum exists in $P$. 
Definition 2.8. Let $(X, \sqsubseteq, d)$ be an ordered cone metric space with cone $P$, $k$ a positive integer and $T: X^{k} \rightarrow X$ be a mapping. Then the mapping $T$ is called a weakly ordered $\phi$ - $\psi$-Prešić type contraction if there exist $\phi \in \Phi(P, C)$, $\psi \in \Psi(B)$ and $\lambda \in(0,1)$ such that

$$
\begin{gathered}
\phi\left[d\left(T\left(x_{1}, x_{2}, \ldots, x_{k}\right), T\left(x_{2}, x_{3}, \ldots, x_{k+1}\right)\right)\right] \\
\preceq_{\mathrm{C}} \lambda \psi\left(\phi\left[d\left(x_{1}, x_{2}\right)\right], \phi\left[d\left(x_{2}, x_{3}\right)\right], \ldots, \phi\left[d\left(x_{k}, x_{k+1}\right)\right]\right)
\end{gathered}
$$

for all $x_{1}, x_{2}, \ldots, x_{k+1} \in X$ with $x_{1} \asymp x_{2} \asymp \cdots \asymp x_{k+1}$.

Remark 2.3. Note that, a weakly ordered Ćirić-Prešić type contraction generalizes the weakly ordered Prešić type contraction (see [2]). Also, for any cone metric space $(X, d)$ with normal cone $P$, normal constant $K$ and a positive integer $k$, define $\phi: P \rightarrow P$ by $\phi[a]=a$ for all $a \in P$ and $\psi\left(x_{1}, x_{2}, \ldots, x_{k}\right)=$ $\max \left\{x_{1}, x_{2}, \ldots, x_{k}\right\}$ for all $x_{1}, x_{2}, \ldots, x_{k} \in P$ (provided the maximum exists in $P)$. Then $\phi \in \Phi(P, C)$ and $\psi \in \Psi(B)$ with $E=B, P=C$ and $\alpha=1$ and the weakly ordered $\phi$ - $\psi$-Prešić type contraction reduced in to Ćirić-Prešić type contraction.

Now we can state our main results.

\section{Fixed point theorems}

The following theorem provides a sufficient condition for the existence of fixed point of a weakly ordered $\phi$ - $\psi$-Prešić type contraction in ordered cone metric spaces.

Theorem 3.1. Let $(X, \sqsubseteq, d)$ be an ordered complete cone metric space with normal cone $P$. Let $k$ be a positive integer and $T: X^{k} \rightarrow X$ be a mapping such that the following conditions hold:

(a) $T$ is a weakly ordered $\phi$ - $\psi$-Prešić type contraction, that is, satisfies $(2.3)$;

(b) $T$ is weakly monotone with respect to $\sqsubseteq$;

(c) there exist $x_{1}, x_{2}, \ldots, x_{k} \in X$ such that $x_{1} \asymp x_{2} \asymp \cdots \asymp x_{k} \asymp T\left(x_{1}, x_{2}\right.$, $\left.\ldots, x_{k}\right)$ and $\mu=\max \left\{\frac{\phi\left[d\left(x_{1}, x_{2}\right)\right]}{\delta}, \frac{\phi\left[d\left(x_{2}, x_{3}\right)\right]}{\delta^{2}}, \ldots, \frac{\phi\left[d\left(x_{k}, T\left(x_{1}, x_{2}, \ldots, x_{k}\right)\right)\right]}{\delta^{k}}\right\}$ exists in $C$ where $\delta=\lambda^{\frac{1}{k}}$;

(d) if a weakly monotone sequence $\left\{x_{n}\right\}$ converges to $x \in X$, then $x_{n} \asymp x$ for all $n \in \mathbb{N}$.

Then $T$ has a fixed point $u \in X$.

Proof. Starting with given $x_{1}, x_{2}, \ldots, x_{k}$ we construct a weakly monotone sequence $\left\{x_{n}\right\}$ in $X$ as follows: since $x_{1}, x_{2}, \ldots, x_{k} \in X$ are such that $x_{1} \asymp x_{2} \asymp$ $\cdots \asymp x_{k}$ and $x_{k} \asymp T\left(x_{1}, x_{2}, \ldots, x_{k}\right)$, let

$$
x_{n+k}=T\left(x_{n}, x_{n+1}, \ldots, x_{n+k-1}\right) \text { for all } n \in \mathbb{N} \text {. }
$$

Therefore $x_{1} \asymp x_{2} \asymp \cdots \asymp x_{k} \asymp x_{k+1}$ and $T$ is weakly monotone with respect to " $\sqsubseteq$ " it implies that $T\left(x_{1}, x_{2}, \ldots, x_{k}\right) \asymp T\left(x_{2}, x_{3}, \ldots, x_{k+1}\right)$, that is, $x_{k+1} \asymp$ 
$x_{k+2}$. Continuing this process we obtain:

$$
x_{1} \asymp x_{2} \asymp \cdots \asymp x_{k} \asymp x_{k+1} \asymp \cdots \asymp x_{n} \asymp x_{n+1} \asymp \cdots .
$$

Thus $\left\{x_{n}\right\}$ is a weakly monotone sequence in $X$. We shall prove that $\left\{x_{n}\right\}$ is a Cauchy sequence in $X$. For simplicity set $d_{n}=d\left(x_{n}, x_{n+1}\right)$ for all $n \geq 0$.

By the method of mathematical induction we shall prove that

$$
\phi\left[d_{n}\right] \preceq_{\mathrm{C}} \mu \delta^{n} \text { for all } n \in \mathbb{N} .
$$

By definition of $\mu$ it is clear that (3.1) is true for $n=1,2, \ldots, k$. Let the $k$ inequalities

$$
\phi\left[d_{n}\right] \preceq_{\mathrm{C}} \mu \delta^{n}, \phi\left[d_{n+1}\right] \preceq_{\mathrm{C}} \mu \delta^{n+1}, \ldots, \phi\left[d_{n+k-1}\right] \preceq_{\mathrm{C}} \mu \delta^{n+k-1}
$$

be the induction hypothesis. Since $\left\{x_{n}\right\}$ is a weakly monotone sequence, $\psi \in$ $\Psi(B)$ and $\delta=\lambda^{\frac{1}{k}} \in(0,1)$ so it follows from (2.3) that

$$
\begin{aligned}
\phi\left[d_{n+k}\right] & =\phi\left[d\left(x_{n+k}, x_{n+k+1}\right)\right] \\
& =\phi\left[d\left(T\left(x_{n}, x_{n+1}, \ldots, x_{n+k-1}\right), T\left(x_{n+1}, x_{n+2}, \ldots, x_{n+k}\right)\right)\right] \\
& \preceq_{\mathrm{C}} \lambda \psi\left(\phi\left[d\left(x_{n}, x_{n+1}\right)\right], \phi\left[d\left(x_{n+1}, x_{n+2}\right)\right], \ldots, \phi\left[d\left(x_{n+k-1}, x_{n+k}\right)\right]\right) \\
& =\lambda \psi\left(\phi\left[d_{n}\right], \phi\left[d_{n+1}\right], \ldots, \phi\left[d_{n+k-1}\right]\right) \\
& \preceq_{\mathrm{C}} \lambda \psi\left(\mu \delta^{n}, \mu \delta^{n+1}, \ldots, \mu \delta^{n+k-1}\right) \\
& \preceq_{\mathrm{C}} \lambda \psi\left(\mu \delta^{n}, \mu \delta^{n}, \ldots, \mu \delta^{n}\right) \\
& \preceq_{\mathrm{C}} \lambda \mu \delta^{n} \\
& =\mu \delta^{n+k} .
\end{aligned}
$$

Thus the inductive proof of (3.1) is complete.

Let $n, m \in \mathbb{N}$ with $m>n$, then we have

$$
\begin{aligned}
d\left(x_{n}, x_{m}\right) & \preceq_{\mathrm{P}} d\left(x_{n}, x_{n+1}\right)+d\left(x_{n+1}, x_{n+2}\right)+\cdots+d\left(x_{m-1}, x_{m}\right) \\
& =d_{n}+d_{n+1}+\cdots+d_{m-1} .
\end{aligned}
$$

As $\phi \in \Phi(P, C)$, using (3.1) we obtain

$$
\begin{aligned}
\phi\left[d\left(x_{n}, x_{m}\right)\right] & \preceq_{\mathrm{C}} \alpha \phi\left[d_{n}+d_{n+1}+\cdots+d_{m-1}\right] \\
& \preceq_{\mathrm{C}} \alpha \phi\left[d_{n}\right]+\alpha \phi\left[d_{n+1}\right]+\cdots+\alpha \phi\left[d_{m-1}\right] \\
& \preceq_{\mathrm{C}} \alpha \mu \delta^{n}+\alpha \mu \delta^{n+1}+\cdots+\alpha \mu \delta^{m-1} \\
& \prec_{\mathrm{C}} \frac{\alpha \mu \delta^{n}}{1-\delta} .
\end{aligned}
$$

As $\delta \in(0,1)$ we obtain from the above inequality that $\lim _{n, m \rightarrow \infty} \phi\left[d\left(x_{n}, x_{m}\right)\right]=$ $0_{\mathrm{B}}$ and $\phi \in \Phi(P, C)$ we have $\lim _{n, m \rightarrow \infty} d\left(x_{n}, x_{m}\right)=0_{\mathrm{E}}$. Therefore by Lemma 2.2, $\left\{x_{n}\right\}$ is a Cauchy sequence. Since $X$ is complete, there exists $u \in X$ such that

$$
\lim _{n \rightarrow \infty} x_{n}=u \text {. }
$$

We shall show that $u$ is a fixed point of $T$. 
If $u \neq T(u, u, \ldots, u)$, then $0_{\mathrm{E}} \prec d(u, T(u, u, \ldots, u))$ and we have

$$
\begin{array}{rl} 
& d\left(x_{n+k}, T(u, u, \ldots, u)\right) \\
= & d\left(T\left(x_{n}, x_{n+1}, \ldots, x_{n+k-1}\right), T(u, u, \ldots, u)\right) \\
\preceq_{\mathrm{P}} & d\left(T\left(x_{n}, x_{n+1}, \ldots, x_{n+k-1}\right), T\left(x_{n+1}, \ldots, x_{n+k-1}, u\right)\right) \\
& +d\left(T\left(x_{n+1}, \ldots, x_{n+k-1}, u\right), T\left(x_{n+2}, \ldots, x_{n+k-1}, u, u\right)\right) \\
& +\cdots+d\left(T\left(x_{n+k-1}, u, \ldots, u\right), T(u, u, \ldots, u)\right) .
\end{array}
$$

Since $\phi \in \Phi(P, C)$ we have

$$
\begin{aligned}
& \phi\left[d\left(x_{n+k}, T(u, u, \ldots, u)\right)\right] \\
\preceq_{\mathrm{C}} & \alpha \phi\left[d\left(T\left(x_{n}, x_{n+1}, \ldots, x_{n+k-1}\right), T\left(x_{n+1}, \ldots, x_{n+k-1}, u\right)\right)\right] \\
& +\alpha \phi\left[d\left(T\left(x_{n+1}, \ldots, x_{n+k-1}, u\right), T\left(x_{n+2}, \ldots, x_{n+k-1}, u, u\right)\right)\right] \\
& +\cdots+\alpha \phi\left[d\left(T\left(x_{n+k-1}, u, \ldots, u\right), T(u, u, \ldots, u)\right)\right],
\end{aligned}
$$

by (d) we have $x_{n} \asymp u$ for all $n \in \mathbb{N}$, therefore using (2.3) in the above inequality we obtain

$$
\begin{aligned}
& \phi\left[d\left(x_{n+k}, T(u, u, \ldots, u)\right)\right] \\
\preceq_{\mathrm{C}} & \alpha \lambda \psi\left(\phi\left[d_{n}\right], \phi\left[d_{n+1}\right], \ldots, \phi\left[d\left(x_{n+k-1}, u\right)\right]\right) \\
& +\alpha \lambda \psi\left(\phi\left[d_{n+1}\right], \ldots, \phi\left[d\left(x_{n+k-1}, u\right)\right], \phi[d(u, u)]\right)+\cdots \\
& +\alpha \psi\left(\phi\left[d\left(x_{n+k-1}, u\right)\right], \phi[d(u, u)], \ldots, \phi[d(u, u)]\right) .
\end{aligned}
$$

Since $\lim _{n \rightarrow \infty} x_{n}=u$, therefore by normality of cone $P$ we have $\lim _{n \rightarrow \infty} d\left(x_{n}, u\right)$ $=0_{\mathrm{E}}$ and $\phi \in \Phi(P, C)$, so $\lim _{n \rightarrow \infty} \phi\left[d\left(x_{n}, u\right)\right]=0_{\mathrm{B}}$. Thus, for every $c \in C^{0}$ there exists $n_{0} \in \mathbb{N}$ such that $\phi\left[d\left(x_{n}, u\right)\right] \ll_{\mathrm{C}} \frac{c}{\alpha k}, \phi\left[d\left(x_{n}, x_{n+1}\right)\right] \ll_{\mathrm{C}} \frac{c}{\alpha k}$ for all $n>n_{0}$. Also $0_{B} \ll_{\mathrm{C}} \frac{c}{\alpha k}$, so using the fact that $\psi \in \Psi(B)$ and inequality (3.2) we obtain

$$
\begin{aligned}
& \phi\left[d\left(x_{n+k}, T(u, u, \ldots, u)\right)\right] \\
& \preceq_{\mathrm{C}} \alpha \lambda \psi\left(\frac{c}{\alpha k}, \frac{c}{\alpha k}, \ldots, \frac{c}{\alpha k}\right)+\alpha \lambda \psi\left(\frac{c}{\alpha k}, \ldots, \frac{c}{\alpha k}, \frac{c}{\alpha k}\right) \\
&+\cdots+\alpha \lambda \psi\left(\frac{c}{\alpha k}, \frac{c}{\alpha k}, \ldots, \frac{c}{\alpha k}\right) \\
& \preceq_{\mathrm{C}} \alpha \lambda \frac{c}{\alpha k}+\alpha \lambda \frac{c}{\alpha k}+\cdots+\alpha \lambda \frac{c}{\alpha k} \\
&=\quad \frac{\alpha \lambda k}{\alpha k} c \\
& \preceq_{\mathrm{C}} c \text { for all } n>n_{0} .
\end{aligned}
$$

For any given $\varepsilon>0$, using the normality of cone $C$ and choosing $c \in C^{0}$ such that $\|c\|<\frac{\varepsilon}{K}$ (where $K$ is the normal constant of $C$ ) we obtain from the above inequality that $\left\|\phi\left[d\left(x_{n+k}, T(u, u, \ldots, u)\right)\right]\right\|<\varepsilon$ for all $n>n_{0}$. Therefore

$$
\lim _{n \rightarrow \infty} \phi\left[d\left(x_{n+k}, T(u, u, \ldots, u)\right)\right]=0_{\mathrm{B}} .
$$


As $\phi \in \Phi(P, C)$, we have $\lim _{n \rightarrow \infty} d\left(x_{n+k}, T(u, u, \ldots, u)\right)=0_{\mathrm{E}}$ and $\lim _{n \rightarrow \infty} x_{n}$ $=u$ therefore by Lemma 2.2 we obtain

$$
d(u, T(u, u, \ldots, u))=0_{\mathrm{E}} .
$$

This contradiction shows that $T(u, u, \ldots, u)=u$. Thus $u$ is a fixed point of $T$. This completes the proof.

Example 3.1. Let $E=\mathbb{R}^{2}$ with Euclidean plane, and $P=\left\{(a, b) \in \mathbb{R}^{2}: a, b \geq\right.$ $0\}$, then $P$ is a normal cone in $E$ with normal constant $K=1$. Let $X=\mathbb{R}^{2}$ and $x_{1}=\left(a_{1}, b_{1}\right), x_{2}=\left(a_{2}, b_{2}\right) \in X$, then the mapping $d: X \times X \rightarrow E$ be defined by

$$
d\left(x_{1}, x_{2}\right)=\left(\left|a_{1}-a_{2}\right|,\left|b_{1}-b_{2}\right|\right)
$$

is a cone metric on $X$ and $(X, d)$ is a complete cone metric space. Define a partial order $\sqsubseteq$ on $X$ by

$$
\begin{aligned}
\left(a_{1}, b_{1}\right) \sqsubseteq\left(a_{2}, b_{2}\right) \Longleftrightarrow & \left(a_{1} \leq a_{2}, b_{1} \leq b_{2} \text { with } a_{1}, a_{2}, b_{1}, b_{2} \in[0,1)\right) \\
& \text { or }\left(a_{1} \leq a_{2}, b_{1} \leq b_{2} \text { with } a_{1}, a_{2}, b_{1}, b_{2} \in[1,2)\right) \\
& \text { or }\left(a_{1}=a_{2}, b_{1}=b_{2} \text { for all } a_{1}, a_{2}, b_{1}, b_{2} \in \mathbb{R}\right) .
\end{aligned}
$$

Let $B=\left\{a x+b: a, b \in \mathbb{R}, x \in\left[\frac{1}{2}, 1\right]\right\}$ be the real vector space with supremum norm and $C=\{a x+b \in E, a \leq 0, b \geq 0\}$ be a normal cone in $B$ with normal constant $K>2$. Define $\phi: P \rightarrow C$ by

$$
\phi[(a, b)]=-a x+b \quad \text { for all }(a, b) \in P .
$$

Then $\phi \in \Phi(P, C)$ with $\alpha=1$. For integer $k=2$, define $\psi: B^{2} \rightarrow B$ by: for all $a_{1} x+b_{1}, a_{2} x+b_{2} \in B$

$$
\psi\left(a_{1} x+b_{1}, a_{2} x+b_{2}\right)=\frac{1}{3}\left[\left(a_{1} x+b_{1}\right)+\left(a_{2} x+b_{2}\right)\right] .
$$

Then $\psi \in \Psi(B)$. For $x_{1}=\left(a_{1}, b_{1}\right), x_{2}=\left(a_{2}, b_{2}\right) \in X$, define a mapping $T: X^{2} \rightarrow X$ by

$$
T\left(x_{1}, x_{2}\right)= \begin{cases}\frac{1}{7}\left(a_{1}+a_{2}, b_{1}+b_{2}\right) & \text { if } x_{1}, x_{2} \in[0,1) \times[0,1) \\ \frac{1}{7}\left(\frac{1}{a_{1}+a_{2}}, \frac{1}{b_{1}+b_{2}}\right) & \text { if } x_{1}, x_{2} \in[1,2) \times[1,2) \\ \left(\frac{a_{1}}{2}+a_{2}-1, \frac{b_{1}}{2}+b_{2}-1\right) & \text { otherwise }\end{cases}
$$

Then $T$ is a weakly ordered $\phi-\psi$-Prešic type contraction with constant $\lambda=\frac{3}{7}$. Note that all the conditions of Theorem 3.1 are satisfied and $T$ has two fixed points, namely $(0,0)$ and $(2,2)$.

Note that $T$ is not a monotone mapping, that is, $T$ is neither monotone increasing nor monotone decreasing therefore results of [14] are not applicable.

In the above example we see that the fixed point of a weakly ordered $\phi-\psi$ Prešić type contraction may not be unique. In the next theorem we give some sufficient conditions for the uniqueness of fixed point of mapping satisfying the conditions of Theorem 3.1. 
Theorem 3.2. Let $(X, \sqsubseteq, d)$ be an ordered complete cone metric space with normal cone $P$. Let $k$ be a positive integer and $T: X^{k} \rightarrow X$ be a mapping such that all the conditions of Theorem 3.1 are satisfied, then $T$ has a fixed point $u \in X$. In addition, suppose that

(a) the inequality

$$
\phi[d(T(u, u, \ldots, u), T(v, v, \ldots, v))] \prec_{C} \phi[d(u, v)]
$$

holds for all comparable $u, v \in X$ with $u \neq v$, or

(b) in condition $(2.3)$ the constant $\lambda \in\left(0, \frac{1}{\max \{1, k \alpha\}}\right)$.

Then the set of fixed point $\mathcal{F}(T)$ (say) of $T$ is singleton if and only if $\mathcal{F}(T)$ is well-ordered.

Proof. The existence of the fixed point $u \in X$ follows from Theorem 3.1. For uniqueness, suppose that the set of all fixed points of $T$ that is $\mathcal{F}(T)$ is wellordered and (a) is satisfied. Let if possible $v \in \mathcal{F}(T)$ and $u \neq v$. Then as $\mathcal{F}(T)$ is well-ordered it follows from (3.3) that

$$
\phi[d(u, v)]=\phi[d(T(u, u, \ldots, u), T(v, v, \ldots, v))] \prec_{\mathrm{C}} \phi[d(u, v)] .
$$

This is a contradiction. Therefore the fixed point of $T$ is unique.

Suppose that the set of all fixed points of $T$ that is $\mathcal{F}(T)$ is well-ordered and

(b) is satisfied. Again, if $v \in \mathcal{F}(T)$ and $u \neq v$, then we have

$$
\begin{array}{rl}
d(u, v)= & d(T(u, u, \ldots, u), T(v, v, \ldots, v)) \\
\preceq_{\mathrm{P}} & d(T(u, u, \ldots, u), T(u, \ldots, u, v)) \\
& +d(T(u, \ldots, u, v), T(u, \ldots, u,, v, v)) \\
& +\cdots+d(T(u, v, \ldots, v), T(v, v, \ldots, v))
\end{array}
$$

and $\phi \in \Phi(P, C)$ we obtain

$$
\begin{aligned}
\phi[d(u, v)] \preceq_{\mathrm{C}} & \alpha \phi[d(T(u, u, \ldots, u), T(u, \ldots, u, v))] \\
& +\alpha \phi[d(T(u, \ldots, u, v), T(u, \ldots, u, v))] \\
& +\cdots+\alpha \phi[d(T(u, v, \ldots, v), T(v, v, \ldots, v))] .
\end{aligned}
$$

As $\psi \in \Psi(B), \mathcal{F}(T)$ is well-ordered it follows from (2.3) and the above inequality that

$$
\begin{aligned}
\phi[d(u, v)] \preceq_{\mathrm{C}} & \alpha \lambda \psi(\phi[d(u, u)], \ldots, \phi[d(u, u)], \phi[d(u, v)]) \\
& +\alpha \lambda \psi(\phi[d(u, u)], \ldots, \phi[d(u, v)], \phi[d(v, v)]) \\
& +\cdots+\alpha \lambda \psi(\phi[d(u, v)], \phi[d(v, v)], \ldots, \phi[d(v, v)]) \\
\preceq_{\mathrm{C}} & \alpha \lambda \phi[d(u, v)]+\alpha \lambda \phi[d(u, v)]+\cdots+\alpha \lambda \phi[d(u, v)] \\
= & k \alpha \lambda \phi[d(u, v)] .
\end{aligned}
$$

By the choice of $\lambda$ we have $k \alpha \lambda<1$, therefore by Remark 2.1 we have $\phi[d(u, v)]=0_{\mathrm{B}}$, that is, $d(u, v)=0_{\mathrm{E}}$ or $u=v$. Therefore fixed point of $T$ is unique. In both the cases validity of converse is obvious. This completes the proof. 
Following corollary is a generalization and extension of results of [14] for weakly monotone mappings.

Corollary 3.3. Let $(X, \sqsubseteq, d)$ be an ordered complete cone metric space with normal cone $P$. Let $k$ be a positive integer and $T: X^{k} \rightarrow X$ be a mapping such that the following conditions hold:

(a) $T$ is a weakly ordered Prešić-Ćirić type contraction, that is, satisfies $(2.2)$

(b) $T$ is weakly monotone with respect to $\sqsubseteq$;

(c) there exist $x_{1}, x_{2}, \ldots, x_{k} \in X$ such that $x_{1} \asymp x_{2} \asymp \cdots \asymp x_{k} \asymp T\left(x_{1}, x_{2}\right.$, $\left.\ldots, x_{k}\right)$ and $\mu=\max \left\{\frac{d\left(x_{1}, x_{2}\right)}{\delta}, \frac{d\left(x_{2}, x_{3}\right)}{\delta^{2}}, \ldots, \frac{d\left(x_{k}, T\left(x_{1}, x_{2}, \ldots, x_{k}\right)\right)}{\delta^{k}}\right\}$ exists in $P$, where $\delta=\lambda^{\frac{1}{k}}$;

(d) if a weakly monotone sequence $\left\{x_{n}\right\}$ converges to $x \in X$, then $x_{n} \asymp x$ for all $n \in \mathbb{N}$.

Then $T$ has a fixed point $u \in X$.

Proof. Define $\phi: P \rightarrow P$ by $\phi[a]=a$ for all $a \in P$ and $\psi: P^{k} \rightarrow P$ by $\psi\left(x_{1}, x_{2}, \ldots, x_{k}\right)=\max \left\{x_{1}, x_{2}, \ldots, x_{k}\right\}$ for all $x_{1}, x_{2}, \ldots, x_{k} \in P$ (provided the maximum exists in $P$ ). Then $\phi \in \Phi(P, C)$ and $\psi \in \Psi(B)$ with $E=B$, $P=C$ and $\alpha=1$. Now the proof follows from Theorem 3.1.

Following corollary is a generalization and extension of results of [9] for weakly monotone mappings.

Corollary 3.4. Let $(X, \sqsubseteq, d)$ be an ordered complete cone metric space with normal cone $P$. Let $k$ be a positive integer and $T: X^{k} \rightarrow X$ be a mapping such that the following conditions hold:

(a) for all $x_{1}, x_{2}, \ldots, x_{x}, x_{k+1} \in X$ with $x_{1} \asymp x_{2} \asymp x_{k} \asymp x_{k+1}$ there exists $\lambda \in(0,1)$ such that

$\left\|d\left(T\left(x_{1}, x_{2}, \ldots, x_{k}\right), T\left(x_{2}, x_{3}, \ldots, x_{k+1}\right)\right)\right\| \leq \lambda \max \left\{\left\|d\left(x_{i}, x_{i+1}\right)\right\|: 1 \leq i \leq k\right\} ;$

(b) $T$ is weakly monotone with respect to $\sqsubseteq$;

(c) there exist $x_{1}, x_{2}, \ldots, x_{k} \in X$ such that $x_{1} \asymp x_{2} \asymp \cdots \asymp x_{k} \asymp T\left(x_{1}, x_{2}\right.$, $\left.\ldots, x_{k}\right)$

(d) if a weakly monotone sequence $\left\{x_{n}\right\}$ converges to $x \in X$, then $x_{n} \asymp x$ for all $n \in \mathbb{N}$.

Then $T$ has a fixed point $u \in X$.

Proof. Define $\phi: P \rightarrow \mathbb{R}$ by $\phi[a]=\|a\|$ for all $a \in P, \psi:[0, \infty)^{k} \rightarrow[0, \infty)$ by $\psi\left(x_{1}, x_{2}, \ldots, x_{k}\right)=\max \left\{x_{1}, x_{2}, \ldots, x_{k}\right\}$ for all $x_{1}, x_{2}, \ldots, x_{k} \in[0, \infty)$, then $\phi \in \Phi(P, C)$ and $\psi \in \Psi(B)$ with $B=\mathbb{R}, C=[0, \infty)$ and $\alpha=K$, where $K$ is the normal constant of $P$. Note that, with these assumptions in Theorem 3.1 
we have

$$
\begin{aligned}
\mu & =\max \left\{\frac{\phi\left[d\left(x_{1}, x_{2}\right)\right]}{\delta}, \frac{\phi\left[d\left(x_{2}, x_{3}\right)\right]}{\delta^{2}}, \ldots, \frac{\phi\left[d\left(x_{k}, T\left(x_{1}, x_{2}, \ldots, x_{k}\right)\right)\right]}{\delta^{k}}\right\} \\
& =\max \left\{\frac{\left\|d\left(x_{1}, x_{2}\right)\right\|}{\delta}, \frac{\left\|d\left(x_{2}, x_{3}\right)\right\|}{\delta^{2}}, \ldots, \frac{\left\|d\left(x_{k}, T\left(x_{1}, x_{2}, \ldots, x_{k}\right)\right)\right\|}{\delta^{k}}\right\},
\end{aligned}
$$

which always exist in $C=\mathbb{R}$. Therefore the proof follows from Theorem 3.1.

Acknowledgment. The authors are thankful to the editor and the referees for their valuable suggestions and guidelines for improving the manuscript.

\section{References}

[1] S. Banach, Sur les opérations dans les ensembles abstraits et leur applications, Fund. Math. 3 (1922), 133-181.

[2] L. B. Ćirić and S. B. Prešić, On Prešić type generalisation of Banach contraction principle, Acta Math. Univ. Comenian. 76 (2007), no. 2, 143-147.

[3] K. Deimling, Nonlinear Functional Analysis, Springer-Verlag, 1985.

[4] M. Fréchet, Sur quelques points du calcul fonctionnel, Rend. Circ. Mat. Palermo 22 (1906), 1-74.

[5] R. George, K. P. Reshma, and R. Rajagopalan, A generalised fixed point theorem of Prešić type in cone metric spaces and application to morkov process, Fixed Point Theory Appl. 2011 (2011), no. 85, 8 pages.

[6] L. Huang and X. Zhang, Cone metric spaces and fixed point theorems of contractive mappings, J. Math. Anal. Appl. 332 (2007), no. 2, 1468-1476.

[7] G. Jungck, S. Radenović, S. Radojević, and V. Rakočević, Common fixed point theorems for weakly compatible pairs on cone metric spaces, Fixed Point Theory Appl. 2009 (2009), Article ID 643840, 13 pages.

[8] M. S. Khan, M. Berzig, and B. Samet, Some convergence results for iterative sequences of Prešić type and applications, Adv. Difference Equ. 2012 (2012), no. 38, 12 pages.

[9] M. S. Khan and M. Samanipour, Prešić type extension in cone metric space, Int. J. Math. Anal. 7 (2013), no. 36, 1795-1802.

[10] Đ. R. Kurepa, Tableaux ramifiés d'ensembles espaces pseudo-distanciés, C. R. Acad. Sci. Paris 198 (1934), 1563-1565.

[11] - Free power or width of some kinds of mathematical structure, Publ. Inst. Math. (Beograd) 42(56) (1987), 3-12.

[12] S. Lin, A common fixed point theorem in abstract spaces, Indian J. Pure Appl. Math. 18 (1987), no. 8, 685-690.

[13] N. V. Luong and N. X. Thuan, Some fixed point theorems of Prešić-Ćirić type, Acta Univ. Apulensis Math. Inform. 30 (2012), 237-249.

[14] S. K. Malhotra, S. Shukla, and R. Sen, A generalization of Banach contraction principle in ordered cone metric spaces, J. Adv. Math. Stud. 5 (2012), no. 2, 59-67.

[15] - Some coincidence and common fixed point theorems in cone metric spaces, Bull. Math. Anal. Appl. 4 (2012), no. 2, 64-71.

[16] J. J. Nieto and R. R. López, Contractive mapping theorems in partially ordered sets and applications to ordinary differential equation, Order 22 (2005), no. 3, 223-239.

[17] M. Păcurar, Approximating common fixed points of Prešić-Kannan type operators by a multi-step iterative method, An. Şt. Univ. Ovidius Constanţa Ser. Mat. 17 (2009), no. $1,153-168$ 
[18] _ A multi-step iterative method for approximating common fixed points of PrešićRus type operators on metric spaces, Studia Univ. Babeş-Bolyai Math. 55 (2010), no. $1,149-162$.

[19] _ Common fixed points for almost Prešić type operators, Carpathian J. Math. 28 (2012), no. 1, 117-126.

[20] S. Prešić, Sur la convergence des suites, C. R. Acad. Paris 260 (1965), 3828-3830.

[21] A. C. M. Ran and M. C. B. Reurings, A fixed point theorem in partially ordered sets and some application to matrix equations, Proc. Amer. Math. Soc. 132 (2004), no. 5, $1435-1443$.

[22] S. Rezapour and R. Halbarani, Some notes on the paper, Cone metric spaces and fixed point theorem of contractive mappings, J. Math. Anal. Appl. 345 (2008), no. 2, 719-724.

[23] B. Rzepecki, On fixed point theorems of Maia type, Publ. Inst. Math. (Beograd) 28(42) (1980), 179-186.

[24] S. Shukla, Prešić type results in 2-Banach spaces, Afr. Mat. 25 (2014), no. 4, 1043-1051.

[25] S. Shukla and B. Fisher, A generalization of Prešić type mappings in metric-like spaces, J. Oper. 2013 (2013), Article ID 368501, 5 pages.

[26] S. Shukla and S. Radenović, A generalization of Prešić type mappings in 0-complete ordered partial metric spaces, Chinese J. Math. 2013 (2013), Article ID 859531, 8 pages.

[27] S. Shukla and R. Sen, Set-valued Prešić-Reich type mappings in metric spaces, Rev. R. Acad. Cienc. Exactas, Fís. Nat. Ser, A. Mat. 108 (2014), no. 2, 431-440.

[28] S. Shukla, R. Sen, and S. Radenović, Set-valued Prešić type contraction in metric spaces, An. Ştiinţ. Univ. Al. I. Cuza Iaşi. Mat. (N.S.). http://dx.doi.org/10.2478/aicu-20140011.

[29] P. Zabreiko, K-metric and K-normed spaces: survey, Collect. Math. 48 (1997), no. 4-6, $825-859$.

Mohammad SAEed KHAN

Department of Mathematics and Statistics

COllege OF SCIENCE

Sultan Qaboos University

Post Box 36, Postal Code 123, Al-Khod, Muscat, Oman

E-mail address: mohammad@squ.edu.om

SATiSh SHUKLA

Department of Applied Mathematics

Shri Vaishnav Institute of Technology \& Science

Gram Baroli, Sanwer RoAd, Indore (M.P.) 453331, India

E-mail address: satishmathematics@yahoo.co.in

Shin Min Kang

DePartment of MATHEMATICS AND RINS

Gyeongsang National University

JinJu 660-701, Korea

E-mail address: smkang@gnu.ac.kr 